\title{
Pyruvate Kinase Muscle Isozyme Measurement
}

National Cancer Institute

\section{Source}

National Cancer Institute. Pyruvate Kinase Muscle Isozyme Measurement. NCI

Thesaurus. Code C156530.

The determination of the amount of pyruvate kinase muscle isozyme present in a sample. 